\title{
Fiber Bragg grating sensors for spatially resolved measurements in ex-vivo pancreatic laser ablation
}

Daniele Tosi, Abzal Adilzhan, Kamilla Aliakhmet, Paola Saccomandi, Emiliano Schena, Sergio Silvestri, Dinesh Babu Duraibabu, Sven Poeggel, Gabriel Leen, Elfed Lewis

- Department of Electrical and Electronical Engineering

- Biosensors and Boinstruments Laboratory

\begin{abstract}
Laser ablation (LA) is an emerging technology for mini-invasive treatment of tumors, and operates by damaging cancer cells by means of focused light-induced selective heating. In-situ temperature monitoring, with micro-sensors, may be particularly beneficial for both estimating in real-time the LA efficacy, and build prediction and controls for LA procedures. In this paper, we present our latest results on spatially resolved temperature monitoring, based on fiber Bragg grating (FBG). We used in-line FBG arrays based on 6 and 35 FBGs to achieve spatially resolved thermal sensing in LA applied to porcine pancreas. The main experimental results and their implications are hereby discussed.
\end{abstract}

Original language

Title of host publication

Publisher

ISBN (Electronic)

State

Event
English

2016 IEEE International Symposium on Medical Measurements and Applications, MeMeA 2016 - Proceedings

$\underline{\text { Institute of Electrical and Electronics Engineers Inc. }}$

9781467391726

Published - Aug 42016

11th IEEE International Symposium on Medical Measurements and Applications, MeMeA 2016 - Benevento, Italy

Tosi, D., Adilzhan, A., Aliakhmet, K., Saccomandi, P., Schena, E., Silvestri, S., ... Lewis, E. (2016). Fiber Bragg grating sensors for spatially resolved measurements in ex-vivo pancreatic laser ablation. In 2016 IEEE International Symposium on Medical Measurements and Applications, MeMeA 2016 Proceedings. [7533806] Institute of Electrical and Electronics Engineers Inc.. DOI: $\underline{10.1109 / M e M e A .2016 .7533806}$ 